\title{
Teoría e historia. \\ (A propósito de historiografía \\ reciente sobre Nicaragua)*
}

Antonio Acosta Rodríguez

Universidad de Sevilla

Tomando como referencia algunos de los libros publicados recientemente sobre historia de Nicaragua, se pone de manifiesto cómo, con frecuencia, los historiadores utilizan términos tomados en préstamo de otras ciencias sociales, como la sociología, sin precisar su significado y el contexto teórico en que han sido definidos, lo cual varía según las diversas corrientes de esta disciplina durante el presente siglo. Esto, que se presenta como un problema para el avance de la ciencia histórica en la actualidad en el terreno de la teoría, es particularmente grave en una especialidad como la denominada historia social -aún admitiendo las diversas concepciones de esta expresión-, por contraste con lo que sucede en otras, como podría ser la historia económica.

Hace ya años Pierre Vilar, con la brillantez de los maestros, comparaba el comercio de la historia con el de los detergentes. Afirmaba que en el primero las marcas están peor defendidas que en el segundo ya que, a diferencia de lo que sucedía con los detergentes, cualquiera puede llamarse historiador, cuando en realidad "nada hay más difícil y raro que ser historiador, salvo ser historiador marxista". ${ }^{1}$

Lo que podía parecer una boutade de Vilar no lo era en absoluto y con la fantástica profusión historiográfica de las últimas décadas se han sucedido innumerables ejemplos de trabajos sobre todas las épocas y regiones del mundo que ilustran perfectamente la conclusión del gran hispanista francés. Vilar encuadraba su frase en un trabajo desde el que, entre otros aspectos del debate con L. Althusser sobre la concepción estructuralista del marxismo, reclamaba una historia - marxista, por cierto - científica, seria y rigurosamente concebida, desde la selección y el manejo de las fuentes hasta la utilización escrupulosa y dialéctica de un utillaje conceptual cuidadosamente seleccionado y elaborado.

*Algunas de las reflexiones expresadas en este artículo surgieron en los cursos de la Maestría de Historia de la Universidad Centroamericana (Managua-Nicaragua), en 1993-1994. Agradezco a mis estudiantes en dicha Maestría lo estimulante que me resultaron la relación con ellos y las discusiones en las clases.

1 Vilar, P.: "Historia marxista, historia en construcción”, en Hacer la historia. Dirección de J. Le Goff y P. Nora. Barcelona, 1984, vol. I, pág. 179. (La edición en francés, 1974). 
Es ocioso decir que a lo largo del siglo la historia ha hecho avances extraordinarios en la consecución de su objetivo científico. Particularmente en algunas disciplinas o especialidades históricas los esfuerzos por depurar la crítica de las fuentes, el manejo de los datos y las técnicas de análisis, así como el utillaje teórico, han sido enormes y han producido fructíferos debates y progresos en el conocimiento del pasado. Como consecuencia de ello se ha llegado a una situación en que los investigadores de cualquier tendencia ideológica o adscritos a cualquier escuela metodológica se afanan, en general, por ajustarse a los avances conseguidos haciendo más rigurosos sus trabajos.

Esto sucede por ejemplo en la historia económica, que ha experimentado una espectacular progresión en todos los terrenos señalados desde el primer cuarto de siglo. Resultaría imposible reflejar en un breve artículo los importantes logros alcanzados en los últimos decenios, aunque nos refiriésemos sólo a una parcela como la de la historia económica de América Latina. Así, en nuestros días, es raro ver que un historiador que incursione en el estudio de fenómenos económicos no procure intentar cuantificar sus afirmaciones, emplee mal la estadística, o no maneje con un mínimo de soltura y cuidado las relaciones existentes entre las diferentes esferas de lo económico y los conceptos inherentes a aquéllas dependiendo, según los casos, de su posición teórica o ideológica.

Cuando alguna operación en cualquiera de estos terrenos está realizada a la ligera el hecho salta inmediatamente a la vista, llama la atención; hasta tal grado se está familiarizado hoy día con un modus operandi ortodoxo, según los avances de la disciplina, y esto sucede - hay que insistirmás allá de cuál sea la posición ideológica y/o metodológica del autor.

Sin embargo, como "de todo hay en la viña del Señor", a pesar del camino recorrido se pueden citar ejemplos de investigaciones poco cuidadosas en el sentido expresado. Para ilustrar lo que exponemos —en este solo ejemplo no nos referiremos a la historia de Nicaragua-, tomemos el caso del matrimonio estadounidense Richard y Linda Salvucci, quienes abordaron en un trabajo reciente el cálculo de la "renta nacional" de México en torno a $1800 .{ }^{2}$ El problema, en el caso del matrimonio Salvucci, no se encuentra exactamente en la manipulación de los datos aunque, como se apreciará, la simpleza de la información seleccionada constituye de

2 Salvucci, R. y L.: "Las consecuencias económicas de la independencia mexicana", en Leandro Prados y Samuel Amaral (eds.): La Independencia americana: consecuencias económicas. Madrid, 1993, págs. 31-53. 
entrada una barrera difícil de franquear; ni tampoco en la aplicación "en seco" de la teoría económica liberal al México de comienzos del siglo XIX; pero sí en la ejecución precipitada del cálculo de la renta nacional en un territorio enormemente heterogéneo y rico como México, y en una época en que puede dudarse que las fuentes tengan la calidad necesaria como para proceder de forma tan ligera.

Perdónesenos una segunda cita de P. Vilar, pero ya en 1964, en el prólogo de la primera edición de su clásico Crecimiento y desarrollo, hablaba de los peligros que amenazaban a la historia económica y, de entre ellos, el primero procedía

“...de ciertas escuelas de economistas que para demostrar alguna verdad abstracta y elemental, aplican ... tratamientos matemáticos cuyo empleo no llegarían a justificar ni las serias estadísticas más modernas y seguras. Para la estimación de las rentas nacionales y su distribución, resulta aventurado "actualmente" entregarse a ciertos cálculos". ${ }^{3}$

Vilar se refería, naturalmente, a los primeros y controvertidos trabajos de la New Economic History norteamericana y francesa que comenzaban a aparecer a fines de la década de 1950 y comienzos de la de 1960, en los que el cálculo de la renta nacional tenía una importancia clave. Precisamente en 1964 se publicaba uno de los libros pioneros de dicha escuela, obra de Robert W. Fogel, Los ferrocarriles y el crecimiento económico de los Estados Unidos. Ensayos de historia econométrica. ${ }^{4}$ Después de debatir problemas de la historia económica de los Estados Unidos — para la que existen fuentes de una calidad más que aceptable_ en el siglo XIX, Fogel afirmaba

"Que los datos de la historia económica sean frecuentemente parciales no impide la elaboración de pruebas empíricas eficaces para las hipótesis cuantitativas. Otras disciplinas - la arqueología y la paleontología, por ejemplo — han conseguido un gran éxito en el diseño de procedimientos para el aprovechamiento de descubrimientos fragmentarios. Es de un pesimismo gratuito suponer que no pueden alcanzarse éxitos semejantes en la historia económica. No existen normas absolutas sobre exactitud de las mediciones. La amplitud permisible del error en un conjunto dado de estimaciones depende del uso a que se destinen". ${ }^{5}$

3 Vilar, P.: Crecimiento y desarrollo. Barcelona, 1964, pág. 8.

4 Primera edición en inglés bajo el título Railroads and American Economic Growth. Essays in Econometric History. Baltimore, 1964. La primera edición en castellano es de la Editorial Tecnos. Madrid, 1972. R. Fogel fue galardonado en 1993 con el Premio Nobel de Economía.

5 Fogel, R.: Los ferrocarriles..., pág. 233. 
No es de extrañar que trabajos sostenidos por tal tipo de afirmaciones despertasen vivas polémicas y advertencias como la de Vilar, aunque ciertamente la citada obra de Fogel estuviese respaldada por un importante volumen de fuentes. Sin embargo, el matrimonio Salvucci ha ido más allá de las previsiones de Fogel en el trabajo mencionado, incurriendo en los riesgos advertidos por Vilar. Y, en efecto, tras citar esfuerzos realizados anteriormente por otros colegas para evaluar la renta mexicana a fines de la colonia — que dieron resultados tan dispares como 102 y 240 millones de pesos-, se lanzan a calcular el Producto Nacional Bruto de México, tanto por la vía del gasto como por la de la renta, en 1800.

Por no hacer largo el comentario, resumamos brevemente el cálculo efectuado por la vía del gasto. El punto de partida es la estimación efectuada por otro historiador de la "renta de subsistencia per cápita" en México capital a fines del siglo XVIII en 34 pesos al año. Esta estimación es considerada por los autores como más alta que el gasto rural, pese a lo cual es generalizada al conjunto de la población mexicana (5,8 millones de personas, según Humboldt), admitiendo que el resultado desplazará al alza el valor final de la renta. Como si los supuestos implícitos en tal operación no fuesen de por sí arriesgados, el matrimonio Salvucci recuerda que hay que añadir al cálculo la inversión y el gasto público y, a partir de la afirmación de que "los países modernos con renta baja dedican un tercio del PIB al gasto público y a la inversión nacional bruta", deciden que adjudicarle el 10\% al México de 1800 es lo razonable. Así resulta que 5,8 millones de personas x 34 pesos persona/año $=197$ millones de pesos $+10 \%=$ 216 millones. Dado que la cifra se sitúa aproximadamente en el término medio de los cálculos previos de otros historiadores, a los autores les parece que van por el camino correcto.

Veamos ahora el cálculo que hacen por la vía de la renta para comprobar si están en lo cierto. Para empezar, los Salvucci aceptan que en el México de la época "el trabajador rural ganaba una media de 5 pesos al mes y el urbano, 6" (sic). Dando por hecho que el 6\% de los 5,8 millones de habitantes de México hacia 1800 era población urbana, esto es, residiendo en concentraciones de más de 10.000 habitantes, el resto, esto es, el $94 \%$ era población rural, ascendiendo a 5.452.000. A partir de aquí, la operación comienza a ser fácil si uno toma en consideración únicamente que la "edad laboral solía iniciarse a los 15 años, aunque no era infrecuente el empleo de niños más pequeños". Por este motivo, al $60 \%$ de la "población activa", considerando como tal a toda la superior a 15 años, podría añadírsele el 10\% 
del $40 \%$ restante que correspondía a la población menor de 15 años, para tener en cuenta esa participación de menores en el trabajo - es decir, un $4 \%$ del total- Con ello se tiene un $64 \%$ de población activa que, aplicado a los respectivos volúmenes de población rural -3.489.280 personas $\mathrm{x}$ (60 pesos/persona/año) - y urbana - 222.270 personas x (72 pesos/persona/año) - termina arrojando finalmente una cifra de 225 millones de pesos anuales de Producto Interior Bruto en el México de 1800 que pareció verosímil y, por lo tanto, válida a los historiadores estadounidenses.

La operación, en su conjunto, resulta casi espectacular y la extraordinaria ligereza empleada, al simplificar hasta extremos inauditos la economía y la sociedad mexicanas, prácticamente exime de cualquier comentario.

Sin salir de la historia económica, la aplicación a priori de esquemas teóricos - lo que constituye también una ligereza- puede dar lugar igualmente a operaciones arriesgadas de las que resultan trabajos ciertamente llamativos. En este terreno podríamos escoger una obra, ahora sí, sobre Nicaragua para ilustrar cómo salta a la vista un ejercicio ligero y poco fundamentado en historia económica. Fijémonos en el libro de un autor nicaragüense, Oscar René Vargas, quien publicó en 1990 La revolución que inició el progreso (Nicaragua, 1893-1909). ${ }^{6}$ El libro del profesor Vargas es un trabajo de historia económica que pretende demostrar el proceso del surgimiento del capitalismo en Nicaragua en el período de gobierno del general José Santos Zelaya. El problema de estudio abordado en el libro no está mal elegido. No cabe duda de que durante el gobierno de Zelaya ocurrieron cambios de gran importancia que alteraron la estructura económica del país, en general, y debieron afectar a las relaciones de producción, en particular.

$\mathrm{Si}$ alguien estudiase de forma seria especialmente el segundo de estos problemas, sin duda ayudaría a entender la evolución de la sociedad de Nicaragua. ${ }^{7}$ Pero el profesor Vargas no hace eso. Por el contrario, pertrechado fundamentalmente de textos teóricos —en vez de fuentes primarias - y de algunas estadísticas procedentes en su mayor parte de fuentes secundarias, efectúa un recorrido por las claves de la agenda marxista tales como la transición del "precapitalismo" al capitalismo, el estado y las clases sociales..., cruzadas con comentarios en algunos terrenos de la estruc-

6 Vargas, Oscar René: La revolución que inició el progreso. (Nicaragua, 1893-1909). Managua, 1990.

7 Afortunadamente se anuncia un trabajo de la Lic. Margarita Vanini que puede iluminar de manera importante este problema crucial de la historia nicaragüense. 
tura económica como el mercado interno, la industria o el comercio exterior y la influencia extranjera, que recibe el más largo capítulo del libro.

Tratándose de propugnar la aparición del capitalismo, un estudio a fondo de la evolución de las relaciones de producción hubiera sido esencial $\mathrm{y}$, sin embargo, sobre esta clave del problema únicamente se presentan algunas pinceladas inconexas. El asunto se aborda en el punto 3.2 del capítulo III. En una página y a partir exclusivamente de una encuesta de población realizada en 1902-1903 — aunque también se refiere al censo de 1896determina, entre otras conclusiones, que "la mayor parte de la población económicamente activa rural del país se encontraba bajo el régimen de asalariados, aunque fuese por el tiempo de las cosechas" (pág. 80). Esta endeble fundamentación de la existencia de capitalismo en el país se refuerza con afirmaciones como la de que "en el importante desarrollo de la ganadería comercial... el crecimiento de la producción de queso tiene una importancia especial ya que muestra que se está operando un cambio en la mentalidad de los ganaderos, haciéndolos capitalistas" (pág. 89). Esta línea argumental se completa en el capítulo VII dedicado al Estado, las clases sociales... plagado de consideraciones puramente teóricas, etéreas, sin ningún apoyo en la realidad que se supone que se estudia (pág. 219).

Para tratar de demostrar un asunto de tanta envergadura como el que el autor se propone, el libro habría necesitado un acucioso trabajo de archivo que brilla por su ausencia, careciendo, como consecuencia, por completo de un apoyo documental suficiente en el que sustentar la tesis que pretende defender. Como muestra de la falta de apoyo documental primario y suficiente del libro, señalemos sólo a título de ejemplo que el capítulo I contiene 35 notas a pie de página, de las que 26 son citas de Carlos Marx, o que en el capítulo IV la proporción tampoco es despreciable: de 87 notas, 26 corresponden a citas de Marx y Lenin. Sin entrar en más detalles en el comentario del libro que no se ajustarían al objetivo de este artículo, concluyamos diciendo que el resultado del trabajo del profesor Vargas es un producto esclerotizado por haber pretendido encajar como un corsé una teoría rígidamente concebida a una realidad observada únicamente a vista de pájaro, en sus rasgos más gruesos, en lugar de ser lo que debiera realizar un historiador que se proclama marxista: "aplicar un modo de análisis teóricamente elaborado a la más compleja de las materias de ciencia: las relaciones sociales entre los hombres y las modalidades de sus cambios". ${ }^{8}$

8 Vilar, P.: "Historia marxista...". 
Por diferentes motivos, trabajos como los citados, correspondientes a una disciplina como la historia económica, son una muestra de cómo la aplicación ligera o incorrecta de métodos de análisis o planteamientos teóricos llaman rápidamente la atención hoy en día después de los avances que esta especialidad histórica ha experimentado.

Pero si esto es así en historia económica, algo muy diferente sucede en el terreno de la historia social o, si se prefiere, del análisis de las sociedades del pasado. Ciertamente nunca ha terminado de aclararse satisfactoriamente, a gusto de todos o ni siquiera de la mayoría, la expresión "historia social". Diferentes escuelas, tendencias historiográficas y hasta historiadores individuales han efectuado diversas interpretaciones acerca de esta etiqueta. ${ }^{9}$ Por otra parte, el crecimiento historiográfico se ha asociado frecuentemente con la preocupación por estudiar sectores sociales por los que la historia tradicional positivista no se interesaba y, como consecuencia de ello, algunos han llegado a calificar como "nueva historia" la producida con estos intereses. ${ }^{10}$

Sin embargo, aunque no se pretenda rescatar aquí ninguna de las varias definiciones u orientaciones de la disciplina, admitamos al menos la tarea: el análisis de las sociedades del pasado, y situémonos en el ámbito que cualquier historiador tiene que transitar, esto es, el que le lleva a intentar comprender y explicar cómo se compone, funciona y cambia la sociedad que estudia. En este ámbito la situación por la que atraviesa la historia desde hace décadas puede calificarse, sin temor a exageración, de una asumida y gigantesca confusión. Y ello vale, en general, para trabajos que tratan desde la antigüedad hasta el siglo XX y para todos los espacios geográficos.

Lo que con diversos significados se llama historia social, aunque con raíces historiográficas que se remontan al siglo XIX, ha vivido a partir del primer tercio del siglo XX una aproximación creciente a la sociología, que se aceleró después de la II Guerra Mundial y de donde procede gran parte del problema que se pretende reflejar aquí. Esta proximidad fue provocada

\footnotetext{
9 Para un planteamiento general de la cuestión, véase Casanova, Julián: La historia social y los historiadores. Barcelona, 1991.

10 La calificación de "nueva historia" ha dado lugar incluso a una monografía de la historiadora norteamericana Himmelfarb, Gertrude: The New History and the Old. Cambridge, Mass., 1987. Según la autora, la "nueva historia" sería, entre otras cosas, la "historia desde abajo", entendida la expresión con referencia a la escala social, aunque si se la quiere identificar a partir de este enfoque hay que remontarse nada menos que a 1924 para recordar que ya entonces Georges Lefèbvre incorporaba la "perspective d'en bas" en su libro Les paysans du Nord pendant la Revolution Française. Paris, 1924. En 1987, por tanto, esta historia ya no era tan nueva.
} 
principalmente por la evidencia de que la historia no había sido capaz de generar un cuerpo de teoría con cuyos conceptos analizar su objeto de estudio, en este caso las sociedades del pasado. ${ }^{11}$

Sin embargo, este contacto que debería haber proporcionado a la historia los elementos teóricos de los que supuestamente carecía, ciertamente lo ha hecho en muchos casos pero aún en muchísimos más ha ocasionado otro problema igual de grave, si no más, que el que se procuraba solucionar. Y, en efecto, no se exagera si se afirma que en nuestros días una mayoría de historiadores utiliza una gran variedad de conceptos prestados de la sociología, sin consideración a cuál es la teoría que los respalda y sin detenerse, aunque sea brevemente, ni siquiera a definir lo que entienden por ellos.

Expresiones como "clase", "clase(s) alta(s)", "clase(s) media(s)", "clases trabajadoras", "élite(s)" de diferente carácter... son algunas de las manejadas frecuentemente y, a veces, simultáneamente sin que se sepa a ciencia cierta qué contenido social y teórico se les da al usarlas. Por añadidura son usadas antes del correspondiente análisis, con lo que se convierten a priori en una especie de paraguas o escudos protectores que liberan al historiador que los usa de la difícil tarea de diseccionar la sociedad y descubrir, a posteriori, cómo estaba conformada. Estos conceptos y otros han sido definidos y redefinidos por sucesivos autores y corrientes de la sociología, adquiriendo significados específicos en cuerpos teóricos determinados, a pesar de lo cual muchos historiadores los utilizan de forma indiscriminada, con sólo un vago sentido que el lector debe intuir, desnaturalizándose como consecuencia por completo su significado.

Este es uno de los problemas de que adolece la historia social (deberíamos decir historia, a secas) en nuestros días, que convierte a la historiografía en un totum revolutum conceptual, que hace que trabajos que estén técnicamente bien elaborados carezcan de la más mínima solidez teórica, aunque aparenten tenerla. Y ello a pesar de, o quizás a causa de la generalmente poco madura colaboración entre la sociología y la historia. Nos enfrentamos así a un serio problema que sólo muy ocasionalmente ha sido apuntado.

Rastrear con detenimiento su origen exige una investigación que es esencial, pero que se encuentra más allá del alcance de este trabajo y de

11 J. Casanova, por ejemplo, proporciona un resumen aproximativo de esta discusión en $\mathrm{La}$ historia social..., págs. 55-57. 
las posibilidades actuales de quien escribe. Sin embargo, parece importante mostrar al menos la situación del caso, dejando para más adelante la detección de su inicio. Algunos de los estudios publicados en los últimos años sobre historia de Nicaragua sirven perfectamente para ilustrar el asunto. Veamos tres ejemplos con diferentes características en relación con la cuestión.

En primer lugar hay autores que no participan de esta confusión señalada, o mejor dicho, lo hacen desde fuera de cualquier marco teórico, sencillamente porque no se adscriben a ninguna tendencia sociológica ni utilizan ninguno de los múltiples conceptos-herramientas referidos más arriba. El hecho de asumir una concepción específica de la sociedad ya implica de por sí un compromiso y una complicación que muchos historiadores, ya sea por principios metodológicos o por comodidad ideológica, no están dispuestos a afrontar y prefieren dejar que el pasado hable por sí solo.

Así hay autores que, a la hora de proceder a un análisis de las sociedades del pasado, recurren a aquellas categorías sociales que los sujetos estudiados habían acuñado para describirse a sí mismos. De este modo continúan, por así decirlo, en el estadio previo al de la aproximación entre la sociología y la historia. Al margen de que se trata de una opción respetable, como cualquier otra, esta práctica ausencia de teoría presenta algunos inconvenientes. Por una parte, adoptar las categorías sociales de la propia sociedad estudiada y que se encuentran en la documentación histórica conlleva el riesgo de terminar usando la perspectiva de un solo sector de la sociedad para comprender a la sociedad entera, normalmente el sector dominante, aquél que genera dicha documentación. Pero, por otro lado, si todos los historiadores procediesen de esa forma, con tal grado de ausencia de teoría, nos enfrentaríamos a un particularismo generalizado que difícilmente permitiría establecer comparaciones y síntesis de casos. Podemos citar el trabajo de un autor nicaragüense para ilustrar lo que decimos; se trata del profesor Germán Romero y su libro Las estructuras sociales de Nicaragua en el siglo XVIII. ${ }^{12}$

Para el Dr. Romero una clave de la sociedad estudiada es la dominación colonial. Este hecho dio lugar a la existencia de vencidos y vencedores, indígenas y españoles, aunque esta realidad dio paso poco a poco al surgimiento de otro gran componente en la sociedad, resultante de la mis-

12 Romero Vargas, Germán: Las estructuras sociales de Nicaragua en el siglo XVIII. Managua, 1988. 
cigenación entre blancos, indios, más los negros: las llamadas castas, o población ladina en el libro del Dr. Romero, de gran complejidad interna. Así era étnicamente la sociedad colonial.

Por otro lado, el Dr. Romero parte en su estudio de una hipótesis:

"una estratificación social es ante todo un fenómeno subjetivo de la sociedad estudiada - los individuos como se ven unos a otros- perceptible a través del comportamiento de quienes la componían... Los estratos que distinguimos no se encasillan en los marcos preestablecidos por nosotros sino que son la cristalización del comportamiento de los hombres viviendo juntos. Estos estratos se formaban, no según criterios nuestros, sino según juicios de valor, criterios de clasificación social, propios de la sociedad estudiada en un momento dado de su evolución". ${ }^{13}$

Naturalmente esta posición es importante para aproximarse a la visión de la sociedad sobre sí misma y vale especialmente para los vencedores (ver la apreciación de los españoles sobre sí mismos, pág. 67), pero conduce a adoptar como criterios de clasificación social para los vencidos aquéllos acuñados por los primeros, a los que el Dr. Romero denomina "grupo de dominadores" (pág. 171), o "estrato dominante" (págs. 167 y 173). Así, al hablar de la vida comunal y las comunidades, se refiere al "estrato constituido por los indios (cuya estructura interna no escapaba a antagonismos profundos)" (págs. 87 y 105), y cuya composición se entiende en términos de "caciques y principales" por un lado y el "común", por otro (pág. 76). Los primeros "constituían en cierto modo la aristocracia local... descendientes de la antigua aristocracia precolombina" (pág. 374).

Obviamente "caciques", "principales" y el "común" no son criterios de clasificación social acuñados por la sociedad indígena estudiada, como se reclamaba arriba, sino sólo por un sector del conjunto social, precisamente el español. Sabemos, por estudios de otros casos coloniales, que los "dominados" tenían a su vez su propia visión de la sociedad y utilizaban sus propios conceptos para definirse a sí mismos y a los "dominadores". En el caso de Nicaragua, seguramente por problemas de fuentes, esta visión quizás no sea posible reconstruirla, pero en el trabajo del Dr. Romero dicha inquietud ni siquiera se plantea y se da por definitiva la concepción social de los españoles.

Por otra parte, la posición del Dr. Romero, alejada de cualquier esfuerzo de teorización, dificulta, como se adelantó, la comparación con

13 Ibídem, págs. 19-20. 
otros casos y ello se aprecia al abordar el estudio del estrato que se comprime bajo la denominación de "ladinos", "un nuevo estrato en la sociedad colonial... mezcla étnica entre conquistados, conquistadores y esclavos..." (pág. 374). El término "ladinos" fue creado evidentemente por los españoles y abarca un grupo numéricamente en aumento y muy heterogéneo étnicamente que engloba mestizos, mulatos, zambos y otras de las llamadas "castas". Al tratar de comparar este "estrato" condensado con lo que sucedía en otros casos de sociedades coloniales americanas surgen dificultades que evidencian, como se apuntaba, el particularismo de este tipo de estudios.

El libro del Dr. Romero se basa en un excelente trabajo de investigación, sólidamente apoyado en una abundante base documental, pero en el que contrasta su carácter esencialmente descriptivo frente a la citada ausencia de preocupación teórica.

El segundo trabajo que sirve para ilustrar el diverso grado de relación existente entre historia y teoría sociológica es el del historiador estadounidense E. Bradford Burns, Patriarch and Folk. The Emergence of Nicaragua. $1798-1858 .{ }^{14} \mathrm{~B}$. Burns ofrece ya en el título de su libro cuáles son los conceptos claves para comprender la composición de la sociedad nicaragüense de la primera mitad del siglo XIX, justamente la continuación del período estudiado por el Dr. Romero: patriarca y pueblo. Pero ni uno ni otro - bien impreciso el segundo, por cierto - corresponden a ninguna de las escuelas o corrientes dominantes en la sociología contemporánea. ¿Qué pretende decir el autor con ambos términos?

Al comenzar el libro Burns, aunque indirectamente, aclara que el concepto "patriarca(s)" tiene un contenido: se trata de los patriarcas de las "familias dominantes", del "pequeño grupo de comerciantes y terratenientes que anhelaban una visión de prosperidad mediante las exportaciones agrarias..." (pág. 1). Pero, ¿qué lleva a Burns a resumir en el concepto de "patriarca" o "patriarcado" lo que ya se adivina como, al menos, un sector social diversificado de comerciantes y terratenientes?

Para demostrar la naturaleza patriarcal de la sociedad nicaragüense de mediados del XIX, Burns acude a un escándalo en las filas de la "alta sociedad" de León de 1851. El escándalo fue provocado porque el hijo menor del más importante comerciante de la ciudad, Thomas Manning, se había casa-

14 Burns, E. Bradford: Patriarch and Folk. The Emergence of Nicaragua. 1798-l858. Cambridge, Mass., 1991. 
do sin consentimiento paterno atentando contra las costumbres de la época. En medio del conflicto alguien acusó al Sr. Manning de dejarse llevar por "prejuicios de clase" (expresión de Burns), dado que la joven esposa procedía de una "familia proletaria". ${ }^{15}$ Para despejar cualquier posibilidad de duda en el lector sobre si, en efecto, las relaciones entre lo que en el texto se han llamado clases habrían tenido que ver en el problema familiar, Burns se apresura a aclarar:

"Las realidades políticas y económicas derivadas de la lucha de clases amenazaban con complicar la cuestión social. Sin embargo, pese a lo perturbadoras que pudiesen haber sido las implicaciones de la diferencia económica entre los jóvenes esposos, éstas no eran el auténtico problema en la Nicaragua de mediados del siglo XIX". ${ }^{16}$

La aclaración sorprende por un doble motivo. De un lado, por el uso de la expresión y aparente aceptación de la existencia de la lucha de clases - class struggle-, que podría hacer creer que nos encontramos ante un materialista histórico; desde luego, nada más lejos de ello. En segundo lugar, sorprende igualmente el argumento de autoridad: una contundente negativa, en que se basa la eliminación de la importancia de las diferencias económicas (aunque no se especifica de qué naturaleza) como una clave de la sociedad de la época. ${ }^{17}$ Finalmente, Burns fundamenta el concepto de patriarcado en la familia tradicional, cuya existencia explicaría la resistencia a la desintegración de la sociedad nicaragüense en la primera mitad del siglo XIX (pág. 67).

Esta visión casi integrista de la sociedad se diría que aleja aún más a la historia de cualquier esfuerzo teórico, pero el léxico que utiliza Burns en su estudio no se limita a los términos citados, sino que junto a ellos aparecen las expresiones "élites", "élites patriarcales" y "clase alta", entre otras. Elite es un concepto de gran importancia en el desarrollo de la sociología liberal durante el siglo XX. Desde sus primeras definiciones por W. Pareto y G. Mosca a comienzos de siglo, hasta las últimas, de entre las que cabe

15 El entrecomillado es de B. Burns, por lo que hay que suponer que la expresión procede de la fuente. Al acudir a la nota correspondiente en el texto caben dudas de si se trata de Dunlop, Robert: Travel in Central America... London, 1847.

16 Burns, B.: Patriarch..., pág. 66. (la traducción es mía).

17 Esta actitud de B. Burns recuerda al personaje de una tira cómica del dibujante Perich: un niño que — naturalmente hablando de la historia tradicional o, mejor dicho, de la mala historia — explicaba que en clase de historia no sucedía como en la de matemáticas, donde había no sólo que creer en los hechos, sino también demostrarlos; en la de historia, por el contrario, sólo se exigía creerlos. La tira se encuentra en el libro de Fontana, Josep: Historia. Barcelona, 1979. 
destacar la de C.W. Mills, su contenido ha variado sustancialmente. ${ }^{18}$ Este es uno de los términos cuyo uso supuestamente confiere cierta carga de solidez teórica a quien lo utiliza, pero B. Burns es un ejemplo perfecto de los autores que recurren a él sin precisar en absoluto qué entiende cuando lo maneja. No obstante, al avanzar en la lectura del libro y observar la visión extraordinariamente plana y casi idílica que B. Burns ofrece de las comunidades populares, fundamentalmente indígenas (también había mestizos o ladinos, pero a éstos apenas se les presta atención), se diría que el concepto de élite de Burns se aproxima más al de W. Pareto - aún sin ser exactamente aquél - que al de cualquier otro autor posterior. Las mayorías populares, indígenas y mestizos, habrían vivido aisladas en sus propias comunidades (por cierto, también patriarcales), al margen del gobierno central, "manteniendo un prudente equilibrio" (sic) aprendido a lo largo de la experiencia colonial.

Leyendo a B. Burns uno no puede por menos que preguntarse cómo un mundo tan movido y desequilibrado como el que aparece en las páginas de G. Romero se transformó, tras la independencia, en un universo en equilibrio que no se alteraría hasta que los "patriarcas" decidieron copiar al mundo occidental. ¿Desaparecieron de golpe todas aquellas conflictivas relaciones simplemente por el hecho de la independencia política? Es de sospechar que no. Lo más probable es que debido a haber trabajado con fuentes exclusivamente secundarias, por un lado, y a disponer de una concepción simplista de la sociedad, por otro — que no se enriquece aunque se utilicen términos procedentes de la sociología-, B. Burns con su libro se convierte en un paradigma del tipo de problemas que se exponían más arriba en la relación entre sociología e historia.

El tercer caso que merece citarse es la obra de Jeffrey Gould, To lead as equals... ${ }^{19}$ Se trata de un libro en el que se combina trabajo de campo con investigación en archivos y que aborda las transformaciones económicas, políticas e ideológicas en una significativa zona rural de la Nicaragua del siglo XX. A diferencia de los casos anteriores, J. Gould comienza abordando sus planteamientos teóricos. En un apartado dedicado a "Problemas

18 Para una síntesis crítica de la sociología liberal, se puede consultar Laurin-Frenette, Nicole: Las teorías funcionalistas de las clases sociales. Sociología e ideología burguesa. Madrid, 1976. Agradezco a la Lic. Nelly Miranda, de la Universidad Centroamericana (Managua) las provechosas conversaciones mantenidas sobre éste y otros aspectos de la cuestión.

19 Gould, Jeffrey: To lead as equals. Rural Protest and Political Consciousness in Chinandega. Nicaragua, 19l2-1979. Chapel Hill, 1990. 
Metodológicos", Gould empieza por adoptar una determinada definición del concepto originalmente gramsciano de "hegemonía", así como del de "estrategias contrahegemónicas". Y afirma que estos conceptos le ayudaron a comprender la transformación económica, política y cultural en el campo de Chinandega y, en particular, a descifrar las relaciones de la "élite terrateniente" (landed elite) y la de "élite política somocista" con los habitantes de los pueblos de San José y Tonalá (pág. 6). Obviamente el sentido que J. Gould da al concepto de hegemonía no es marxista; de ser así, además, no encajaría con el uso de la expresión "élite", de la que ya tenemos dos manifestaciones: la terrateniente y la política somocista.

Para referirse a los habitantes de San José y Tonalá, J. Gould comenta que dudó en utilizar términos en inglés como rural proletarian, peasant, semiproletarian, peasant laborers, o proletarian que, a su juicio, no captan todas las modalidades de acceso a la tierra y peculiaridades como fuerza de trabajo de dicha población. Finalmente optó por la palabra española "campesino", que le permite referirse al mismo tiempo a comunidades y a organizaciones y que, además, es utilizada por los sujetos que estudia "para describir su propia condición y clase" (pág. 7).

A partir de aquí, Gould se plantea como objetivo "comprender cómo estos campesinos propietarios, aparceros y trabajadores sin tierra, de orígenes geográficos diversos, en menos de una década forjaron un discurso de clase" (pág. 8). A esta altura es necesario preguntarse: ¿a qué tipo de clase social se refiere J. Gould cuando se traza este objetivo? Ciertamente está tratando la relación de los individuos con el medio de producción que es la tierra, y esto aleja lo que parece ser su concepto de clase del de los sociólogos en quien uno pensaría al observar su esbozo teórico: Gerhard Lenski, Raymond Aron o incluso Ralph Dahrendorf. ${ }^{20}$ Pero ya habíamos descartado que estuviera adoptando una aproximación materialista en su estudio; continuemos con su exposición.

El intento de describir este "proceso de formación de una comunidad" — con un discurso de clase - permite a Gould, por otra parte, apreciar que "comunidad y clase no son analíticamente conceptos separados", sino que

"las clases, con raíces en las comunidades, pueden emerger, en términos de relaciones, en oposición a las élites o clases establecidas... Sin considerar sus ‘roles' individuales en las relaciones de producción en el campo, los participantes llegaron a

20 Laurin-Frenette, N.: Las teorías..., Segunda Parte. 
verse a sí mismos como miembros de un grupo social en conflicto contra otro y, de hecho, comenzaron a hablar de su 'clase campesina' en oposición a la ‘clase terrateniente"” (pág. 8).

Una vez más J. Gould hace dudar al lector porque parece que se está ante la expresión práctica de los conceptos marxistas de "clase en sî" y "clase para sî". Para aumentar este pasajero desconcierto Gould regresa poco más adelante al concepto de relaciones de producción y se plantea "¿de qué manera afectaron los cambios técnicos en las relaciones de producción en el Ingenio San Antonio en la conciencia de los trabajadores?" (pág. 14). De todos modos, rápidamente se disipan las dudas sobre su posición ideológica - aunque crecen las teóricas - cuando, en una sola página, comentando los cambios sociales y políticos durante el somocismo, J. Gould habla sucesivamente de "clases trabajadoras", "clases populares", "clases medias", "clases altas" y "élite agraria" (pág. 15).

Sin embargo, a estas alturas surgen otras inquietudes que difícilmente se pueden resolver: ¿qué significan exactamente y qué relación existe entre las expresiones "clases trabajadoras" (ver la explicación del significado del término "trabajador" u "obrero" (en pág. 67) y "clases populares"?, ¿o entre las expresiones "élite agraria", "élite terrateniente", "élite agroexportadora" y "clase terrateniente"?; ¿son sinónimos o marcan diferencias? y, si es así, ¿cuáles? La excelente investigación realizada por J. Gould no resuelve satisfactoriamente estas interrogantes, que más bien se complican al introducirse nuevas expresiones como "oligarquía terrateniente" o "burquesía somocista" (págs. 48 y 213 entre otras). ¿Qué concepción de la sociedad se encierra, finalmente, detrás de tan variopinta terminología?

Como resumen podría decirse que se produce una gran descompensación entre el profundo trabajo de análisis y disección social que se lleva a cabo, tanto en el terreno de las relaciones materiales como en el ideológico por un lado - se trata de un trabajo admirablemente ejecutado desde el punto de vista técnico-, y la traducción teórica que Gould hace del resultado, por otro. Con ella da lugar a un producto conceptualmente confuso que ilustra, quizás mejor que ninguno de los casos anteriormente citados, el problema planteado en este artículo.

No basta con mostrar de entrada al comenzar una investigación una inquietud por la teoría. Más allá que eso, el marco teórico elegido debe ser coherente con el utillaje conceptual que se maneje, ser confrontado a su vez 
con el resultado de la investigación una vez efectuada, y ajustarse a la realidad histórica en la medida de lo necesario, y no al revés. Esta coherencia es la que se encuentra ausente en nuestros días en muchos trabajos de historia social y aún simplemente de historia. Los tres libros comentados en último lugar, que versan desde períodos y enfoques diferentes sobre esta disciplina, son una buena muestra de las dificultades e insatisfactorias relaciones entre teoría e historia. Y, sin embargo, por contraposición a lo que sucede en otras especialidades históricas, estas relaciones apenas son cuestionadas, dándose por aceptables y contribuyendo a la confusión teórica que hemos pretendido poner de relieve. 